Pacific Journal of Mathematics

CHARACTERISTIC POLYNOMIALS OF SYMMETRIC 


\title{
CHARACTERISTIC POLYNOMIALS OF SYMMETRIC MATRICES
}

\author{
EDWARD A. BENDER
}

Let $F$ be a field and $p$ an $F$-polynomial. We say that $p$ is $F$-real if and only if every real closure of $F$ contains the splitting field of $p$ over $F$. Our main purpose is to prove

Theorem 1. Let $F$ be an algebraic number field and $p$ a monic $F$-polynomial with an odd degree factor over $F$. Then $p$ is $F$-real if and only if it is the characteristic polynomial of a symmetric $F$-matrix.

That $p$ must be $F$-real follows from work of Krakowski [4, Satz 3.3]. To prove the coverse we generalize results of Sapiro [6] in Lemma 1 and Theorem 3. Sapiro deals with the case in which $p$ is a cubic. Theorem 4 considers the minimum dimension of symmetric matrices with a given root.

2. A basic lemma. In our proof we shall study congruence classes of certain symmetric matrices which are defined below. We shall denote congruence of the matrices $A$ and $B$ over the field $F$ (i.e., $A=T B T^{\prime}$ for some nonsingular $F$-matrix $T$ ) by $A \sim B(F)$.

Definition. Let $G$ be a field with subfield $F$. If $\lambda \in G$ is nonzero and if $\alpha_{1}, \cdots, \alpha_{n}$ form a basis for $G$ (as a vector space) over $F$, define the matrices $M=\left\|\alpha_{i}^{(j)}\right\|$ and $D(\lambda)=\operatorname{diag}\left(\lambda^{(1)}, \cdots, \lambda^{(n)}\right)$ where superscripts denote conjugacy over $F$. We call

$$
A=A(\lambda)=M D(\lambda) M^{\prime}
$$

a matrix from $G$ to $F$. Clearly

$$
a_{i j}=\operatorname{tr}_{G / F}\left(\lambda \alpha_{i} \alpha_{j}\right) \text {. }
$$

If $\mathscr{A}=\Sigma \oplus G_{i}$ where the $G_{i}$ are extension fields of $F$, and if $A_{i}$ is a matrix from $G_{i}$ to $F$, then any matrix congruent to $\Sigma \oplus A_{i}$ over $F$ is called a matrix from $\mathscr{A}$ to $F$. Note that a different choice for the basis $\alpha_{1}, \cdots, \alpha_{n}$ would lead to a matrix congruent to $A(\lambda)$ over $F$.

Lemma 1. Let $F$ be a field and $p=q_{1} \cdots q_{m}$ a monic $F$-polynomial decomposed into prime factors over $F$. Assume that the splitting field of $p$ over $F$ is a separable extension of $F$. If the identity is a matrix from

$$
\left.\sum_{1}^{m} \oplus F[x] /\left(q_{i}\right)\right)
$$


to $F$, then $p$ is the characteristic polynomial of a symmetric $F$-matrix.

Proof. Let $D=\Sigma \oplus D\left(\lambda_{i}\right)$ and $M=\Sigma \oplus M_{i}$ where the $i^{\text {th }}$ component refers to $F[x] /\left(q_{i}(x)\right)$. We have $T T^{\prime}=M D M^{\prime}$ for some $F$ matrix $T$. Let $E=\Sigma \oplus D\left(\theta_{i}\right)$ where $\theta_{i}$ is a zero of $q_{i}$. By separability $M$ is nonsingular. We have $T^{-1} M D=\left(M^{-1} T\right)^{\prime}$. Let

$$
S=T^{-1}\left(M E M^{-1}\right) T .
$$

Then

$$
\begin{aligned}
S^{\prime} & =\left(M^{-1} T\right)^{\prime} E\left(T^{-1} M\right)^{\prime} \\
& =\left(T^{-1} M\right) E D\left(T^{-1} M\right)^{\prime} \\
& =\left(T^{-1} M\right) E\left(T^{-1} M D\right)^{\prime} \\
& =\left(T^{-1} M\right) E\left(M^{-1} T\right) \\
& =S .
\end{aligned}
$$

Also $|S-\lambda I|=|E-\lambda I|= \pm p(\lambda)$. Finally, $S$ is an $F$-matrix since $M_{i}^{-1}=\left\|\beta_{i}^{(j)}\right\|$ where $\vec{\beta}$ is the complementary basis to $\vec{\alpha}[2$, p. 437].

3. The irreducible case. In this section we shall reduce the proof of Theorem 1 to a study of the prime factors of $p$ over $F$. This requires the Hasse-Minkowski Theorem. The Hilbert symbol over a local field $L$ will be written $(a, b / L)=(a, b)= \pm 1$. If $A$ is a symmetric $L$ matrix and $A \sim \Sigma \oplus a_{i}(L)$, then

$$
c(A / L)=c(A)=\prod_{i \leq j}\left(a_{i}, a_{j}\right)
$$

is the Hasse invariant. If $A$ is a nonsingular symmetric matrix over an algebraic number field $F$, then we have $\operatorname{dim} A$ and $\operatorname{det} A=|A|$ as global invariants, $c\left(A / F_{\mathfrak{p}}\right)$ as Hasse invariants, and $\operatorname{ind}^{+}\left(A / F_{\mathfrak{p}}\right)$ as real archimedean invariants where $\operatorname{ind}^{+}\left(A / F_{\mathfrak{p}}\right)$ is the number of positive $a_{i}$ in $A \sim \Sigma \oplus a_{i}\left(F_{\mathfrak{p}}\right)$.

THEOREM 2. Let $F$ be an algebraic number field and $q$ an $F$-real irreducible $F$ polynomial of degree n. Let $K=F[x] /(q(x))$ and let $k$ be a rational integer.

(1) If $n$ is odd, the identity is a matrix from $K$ to $F$.

(2) If $n$ is even, there is a matrix $A$ from $K$ to $F$ which has the same archimedean invariants as the identity and satisfies $c(A)(|A|,-1)^{k}=+1$ at all local completions of $F$.

The next two sections develop the ideas needed in the proof of this theorem. We now prove Theorem 1 from Lemma 1 and Theorem 2. 
Let $p=q_{1} \cdots q_{s} r_{1} \cdots r_{t}$ be the prime factorization of $p$ over $F$ where the degree $d_{i}$ of $q_{i}$ is odd and the degree $e_{i}$ of $r_{i}$ is even. By assumption $s \neq 0$. Let $A_{i}$ be the matrix from $F[x] /\left(r_{i}(x)\right)$ to $F$ given by Theorem 2 (2) with

$$
k=k(i)=\left(\sum_{j=1}^{i-1} e_{j}+d_{1}-1\right) / 2 .
$$

Let $B_{0}$ be the $d_{1}$ dimensional identity matrix-a matrix from $F[x] /\left(q_{1}(x)\right)$ to $F$ by Theorem 2(1)-and let

$$
B_{i}=\left|A_{i}\right| B_{i-1} \oplus A_{i} .
$$

By induction, the Hasse-Minkowski Theorem gives $B_{i} \sim I(F)$. Thus the identity is a matrix from

$$
F[x] /\left(q_{1}(x)\right) \oplus \sum_{i=1}^{r} \oplus F[x] /\left(r_{i}(x)\right)
$$

to $F$. By Theorem $2(1)$, the identity is a matrix from $F[x] /\left(q_{i}(x)\right)$, so an application of Lemma 1 proves Theorem 1.

4. The local case. In this section we reduce the proof of theorems having the form of Theorem 2 to local considerations.

THEOREM 3. Let $F$ be an algebraic number field and $q$ an F-real irreducible F-polynomial. Let $\alpha_{1}, \cdots, \alpha_{n}$ be algebraic integers in $G=F[x] /(q(x))$ which are a basis for $G$ over $F$. Let $M=\left\|\alpha_{i}^{(j)}\right\|$ and let $Q$ be the set of prime spots on $F$ which divide $2|M|^{2}$. Suppose that for each $\mathfrak{p} \in \Omega$ there is given a matrix $A\left(\lambda_{\mathfrak{p}}\right)$ from $F_{\mathfrak{p}}[x] /(q(x))$ to $F_{\mathfrak{p}}$. Then there is a matrix $A=A(\lambda)$ from $G$ to $F$ and a local prime spot $\mathfrak{q} \notin \Omega$ on $F$ such that

(1) if $\mathfrak{p} \in \Omega$, then

$$
c\left(A / F_{\mathfrak{p}}\right)=c\left(A\left(\lambda_{\mathfrak{p}}\right) / F_{\mathfrak{p}}\right),
$$

and

$$
\left|A\left(\lambda_{\mathfrak{p}}\right)\right| /|A| \in F_{\mathfrak{p}}^{2},
$$

the group of squares in $F_{p}$

(2) if $\mathfrak{p} \notin \Omega$ is a local prime spot on $F$ distinct from $\mathfrak{q}$, then $c\left(A / F_{\mathfrak{p}}\right)=+1$ and $|A|$ is a unit of $F_{p}$;

(3) A has the same real archimedean invariants as the identity matrix of the same dimension.

Proof. If we change the basis used in forming $A\left(\lambda_{\mathfrak{p}}\right)$ and change $\lambda_{\mathfrak{p}}$ by a square factor, then $c\left(A\left(\lambda_{\mathfrak{p}}\right)\right)$ and $\left|A\left(\lambda_{\mathfrak{p}}\right)\right| \cdot F_{\mathfrak{p}}^{2}$ will be unchanged. 
Hence we may assume that $\alpha_{1}, \cdots, \alpha_{n}$ is the basis for all $\mathfrak{p}$ and that $\lambda_{\mathfrak{p}}$ is integral at $\mathfrak{p}$.

There is a sufficiently large positive rational integer $m$ such that

$$
\lambda_{0} \equiv \lambda_{\mathfrak{p}}\left(\bmod \mathfrak{p}^{m}\right) \quad \text { for } \mathfrak{p} \in \Omega
$$

implies

$$
c\left(A\left(\lambda_{0}\right) / F_{\mathfrak{p}}\right)=c\left(A\left(\lambda_{\mathfrak{p}}\right) / F_{\mathfrak{p}}\right) \quad \text { for } \mathfrak{p} \in \Omega
$$

and

$$
\left|A\left(\lambda_{\mathfrak{p}}\right)\right| /\left|A\left(\lambda_{0}\right)\right| \in F_{\mathfrak{p}}^{2} \quad \text { for } \mathfrak{p} \in \Omega \text {. }
$$

Choose $\lambda_{0}$ such that

(i ) $\lambda_{0}$ is an integer of $G$

(ii) $\lambda_{0} \equiv \lambda_{\mathfrak{p}}\left(\bmod \mathfrak{p}^{m}\right)$ for $\mathfrak{p} \in \Omega$

(iii) if $F$ is formally real, $\lambda_{0}$ is totally positive. Let $\mathfrak{M}=\Pi_{\Omega} \mathfrak{p}^{m}$. For each local prime spot $\mathfrak{P}$ on $G$ let $k(\mathfrak{P})$ be the largest rational integer such that $\mathfrak{P}^{k(\mathfrak{P})}$ divides $\lambda_{0}$. Let

$$
\mathfrak{U}=\prod_{\mathfrak{P} \in \Omega} \mathfrak{P}^{k(\mathfrak{P})} \text {. }
$$

Then $\lambda_{0} / \mathfrak{U}$ is prime to $\mathfrak{M}$. By the generalized arithmetic progression theorem [1, Satz 13], there is an $\alpha \in G$ and a prime spot $\mathfrak{D}$ on $G$ such that

(i ) $\left(\alpha \lambda_{0} / \mathfrak{u}\right)=\mathfrak{D}$,

(ii) $\alpha \equiv 1(\bmod \mathfrak{M})$,

(iii) if $F$ is formally real, $\alpha$ is totally positive.

Let $\lambda=\alpha \lambda_{0}$ and let $\mathfrak{q}$ be the prime spot on $F$ which $\mathfrak{D}$ divides. Since $\lambda \equiv \lambda_{0} \equiv \lambda_{\mathfrak{p}}\left(\mathfrak{p}^{m}\right)$, part (1) holds. Since $\lambda$ is totally positive if $F$ is formally real, (3) holds. Since $A(\lambda)$ has integral entries and $|A(\lambda)|=N(\mathfrak{U})|M|^{2}$, a unit of $F_{\mathfrak{p}}$ for $\mathfrak{p} \notin \Omega \bigcup\{\mathfrak{q}\}$, part (2) holds by [5, 92:1].

5. Local lemmas. In this section we prove a series of lemmas. They will be used together with Theorem 3 to prove Theorem 2. Throughout this section we shall let $L$ be a local field with prime spot $\mathfrak{p}$ and characteristic zero; further, $K=K_{1}, K_{2}, \cdots, K_{m}$ will be finite algebraic extensions of $L$.

Lemma 2. If $\mathfrak{p}$ is prime to 2, there is a matrix $A$ from $\Sigma \oplus K_{i}$ to $L$ with integer entries and unit determinant.

Proof. It suffices to exhibit such a matrix from $K$ to $L$. Let $\alpha_{1}, \cdots, \alpha_{n}$ be a free basis for the integers of $K_{i}$ over the integers of $L$. Let $M=\left\|\alpha_{i}^{(j)}\right\|$. The matrix $M^{\prime-1}$ has the form $\left\|\beta_{i}^{(j)}\right\|$ where 
$\beta_{1}, \cdots, \beta_{n}$ is the complementary basis $[2, \mathrm{p} .437]$ to $\alpha_{1}, \cdots, \alpha_{n}$. Let $I I$ be a prime of $K$. The ideal $\left(\beta_{1}, \cdots, \beta_{n}\right)$ equals $\left(\Pi^{k}\right)$ for some rational integer $k$. Since $\left(\alpha_{1}, \cdots, \alpha_{n}\right)=(1)$, there is a matrix $A$, whose elements are integers of $L$ and whose determinant is an $L$ unit, satisfying $M D\left(\Pi^{k}\right)=A M^{\prime-1}$.

For the remainder of this section we shall assume that $\mathfrak{p}$ divides 2.

Lemma 3. If $[K: L]$ is odd, the identity is a matrix from $K$ to L.

Proof. Let $T$ be the inertia subextension of $L$. Suppose that the identity is a matrix from $T$ to $L$, namely $M_{1} D_{1} M_{1}^{\prime}$, and that the identity is a matrix from $K$ to $T$, namely $M_{2} D_{2} M_{2}^{\prime}$. Then the identity is a matrix from $K$ to $L$, namely

$$
\left(M_{1} \otimes M_{2}\right)\left(D_{1} \otimes D_{2}\right)\left(M_{1} \otimes M_{2}\right)^{\prime} .
$$

We first show that the identity is a matrix from $T$ to $L$. Let $M_{1}=$ $\left\|\alpha_{i}^{(j)}\right\|$ where $\alpha_{1}, \cdots, \alpha_{f}$ is a basis for $T$ over $L$. Set $A=M_{1} M_{1}^{\prime}$. Since $T$ is a cyclic extension of $L$, we have $A \sim I(T)$. Since $[T: L]$ is odd, it follows that $A \sim I(L)$.

We now show that the identity is a matrix from $K$ to $T$. Let $\Pi$ be a prime of $K$ such that $\Pi^{e}=\pi$, a prime of $T$, where $e=[K: T]$ is odd. Let $\alpha_{i}=\Pi^{i-1}$ and $M_{2}=\left\|\alpha_{i}^{(j)}\right\|$ and $a=\left(e^{2}-1\right) / 8$. There are two cases.

(i) If $(-1,-1 / T)^{a}=+1$, let $\lambda=1 / e$,

(ii) If $(-1,-1 / T)^{a}=-1$, let

$\lambda=\left(1+\Pi^{-1}+4 \Pi^{-2}\right) / e$.

Set $A=|B| \cdot B$ where $B=M_{2} D(\lambda) M_{2}^{\prime}$. In case (i) it is easily verified that $c(A)=+1$.

We consider case (ii). Since $(-1,-1)^{a}=-1$, it follows that $e \equiv \pm 3(\bmod 8)$. Also, as

$$
-\left(\frac{1-\sqrt{-3}}{2}\right)^{2}-\left(\frac{1+\sqrt{-3}}{2}\right)^{2}=1
$$

we have $f(T(\sqrt{5}) / T)=2$ (see $[5,63: 3]$ ). Thus $(\pi, 5)=-1$ and $(\varepsilon, 5)=+1$ for any unit $\varepsilon$ of $T$. When $e=3$ it is easily shown that $c(A)=+1$. Assume $e>3$. The matrix $B$ has the form shown in Figure I. We shall use the formula $[3$, p. 31]:

$$
c\left(C_{m}\right)=\left(-1,\left|C_{m}\right|\right) \prod_{i=1}^{m-1}\left(\left|C_{i}\right|,-\left|C_{i+1}\right|\right),
$$

if $\Pi_{i=1}^{m}\left|C_{i}\right| \neq 0$, where $C_{i}=\left\|c_{s t}\right\|(1 \leqq s, t \leqq i)$. 


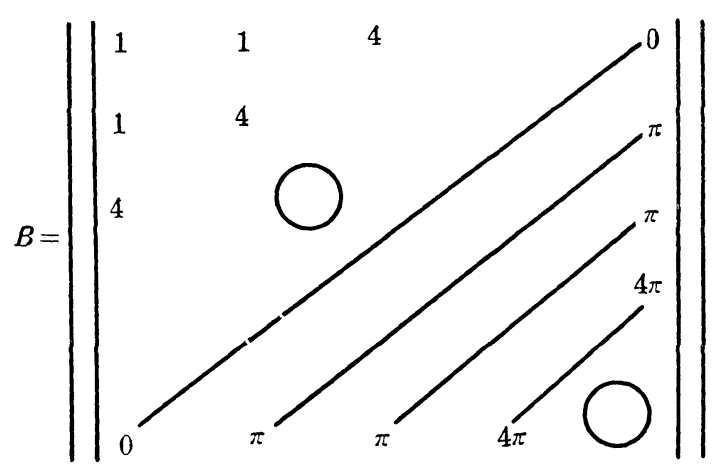

Figure I.

We must transform $B$. Let $X$ be the $e \times e$ matrix such that premultiplication by $X$ adds $\pi^{-1}$ times the $(e-k+2)^{\text {nd }}$ row to the $k^{\text {th }}$ row for $k=4,6,8, \cdots, 4[e / 8]+2$ and leaves the remaining rows unchanged. Let $C=X B X^{\prime}$. By studying $\left(X_{\imath}\right)^{-1} C_{i}\left(X_{i}\right)^{\prime-1}$, we find that

(i) $\left|C_{2 i+1}\right| \in(-1)^{i} T^{2}$ for $2 i+1<e$,

(ii) $\left|C_{e-1}\right| \in(-1)^{(e-1) / 2} 5 T^{2}$,

(iii) $\left|C_{e}\right|=\pi^{e-2} \varepsilon$ for some unit $\varepsilon$ of $T$,

(iv) $\Pi_{1}^{e}\left|C_{i}\right| \neq 0$.

It therefore follows that

(i) $\left(\left|C_{2 i-1}\right|,-\left|C_{2 i}\right|\right)\left(\left|C_{2 i}\right|,-\left|C_{2 i+1}\right|\right)=(-1)^{i-1}$ for $2 i+1<e$,

(ii) $\left(\left|C_{e-2}\right|,-\left|C_{e-1}\right|\right)=(-1)^{(e-3) / 2}$,

(iii) $\left(\left|C_{e-1}\right|,-\left|C_{e}\right|\right)=(-1)^{(e+1) / 2}\left(-1,\left|C_{e}\right|\right)^{(e-1) / 2}$.

Thus

$$
\begin{aligned}
c(A) & =C(B)\left(-1,\left|B_{e}\right|\right)^{(e+1) / 2} \\
& =c\left(C_{e}\right)\left(-1,\left|C_{e}\right|\right)^{(e+1 / 2} \\
& =+1 \quad \text { since } \quad e \equiv \pm 3(\bmod 8)
\end{aligned}
$$

Lemma 4. If $L^{2} \supseteqq N(K / L)$, the norm group of $K$ over $L$, then the identity is a matrix from $K$ to $L$.

Proof. We make some preliminary observations. Let $T_{i}$ be a subfield of $K$ (to be specified later) such that $N\left(K / T_{i}\right) \subseteq T_{i}^{2}$. Let $T_{i}^{*}$ be the multiplicative group of $T_{i}$. Let $H$ be the maximum abelian subextension of $T_{i}$ in $K$ of type $(2,2, \cdots, 2)$. By the reciprocity and limitation theorems of class field theory [7, pp. 177, 180], the Galois group of $H$ over $T_{i}$ is isomorphic to

$$
\left(T_{i}^{*} / N\left(K / T_{i}\right)\right) /\left(T_{i}^{*} / N\left(K / T_{i}\right)\right)^{2} .
$$

Since $N\left(K / T_{i}\right) \subseteq T_{i}^{* 2}$, this is isomorphic to $T_{i}^{*} / T_{i}^{* 2}$. Hence $\left[T_{i}^{*}: T_{i}^{* 2}\right]=$ 
[H: $\left.T_{i}\right]$ which divides $\left[K: T_{i}\right] . \quad$ By $[5,63: 9], 8$ divides $\left[T_{i}^{*}: T_{i}^{* 2}\right] . \quad$ Thus

(i) $\left[K: T_{i}\right] \equiv 0(\bmod 8)$.

Since $N\left(H / T_{i}\right) \subseteq T_{i}^{2}$, we have that $f\left(H / T_{i}\right)>1$. Since $\left[H: T_{i}\right]$ is a power of 2 and $K \supseteqq H$, we have

(ii) $f\left(K / T_{i}\right) \equiv 0(\bmod 2)$.

Suppose $K=T_{i}(\theta)$. Let $\alpha_{i}=\theta^{i-1}$ and $M=\left\|\alpha_{i}^{(j)}\right\|$. If $\lambda \in K$ we have

$$
\left|M D(\lambda) M^{\prime}\right|=N_{K / T_{i}}\left(\lambda \prod_{i \neq 1}\left(\theta^{(1)}-\theta^{(i)}\right)\right) \in T_{i}^{2},
$$

by the formula for a van der Monde determinant and $N\left(K / T_{i}\right) \subseteq T_{i}^{2}$. Hence

(iii) if $C$ is a matrix from $K$ to $T_{i}$, then $|C| \in T_{i}^{2}$.

We now apply the above observations. Let $T$ be the inertia subextension of $L$. Construct the tower

$$
L=T_{0} \subset T_{1} \subset \cdots \subset T_{k} \subseteq T,
$$

where $\left[T_{j}: T_{j-1}\right]=2$ for $1 \leqq j \leqq k$ and $\left[T: T_{k}\right]$ is odd. Since $f\left(K / T_{k}\right)$ is odd, we have $N\left(K / T_{k}\right) \nsubseteq T_{k}^{2}$ by (ii). Hence we may choose $i$ such that $N\left(K / T_{i}\right) \subseteq T_{i}^{2}$ and $N\left(K / T_{i+1}\right) \nsubseteq T_{i+1}^{2}$. (Actually $i=k-1$, but this is irrelevant.) Suppose the identity is a matrix from $K$ to $T_{i}$. Let $B$ be a matrix from $T_{i}$ to $L$. Then $A=I \otimes B$ is a matrix from $K$ to $L$. By (i) we have $\operatorname{dim} I \equiv 0(\bmod 4)$. Hence $|A| \in L^{2}$ and $c(A / L)=+1$ by the formula.

$($ * $) \quad c(X \otimes Y)=c(X)^{y} c(Y)^{x}(-1,|X|)^{y(y-1) / 2}(-1,|Y|)^{x(x-1) / 2}(|X|,|Y|)^{x y+1}$

where $X, Y$ are symmetric matrices, $x=\operatorname{dim} X$ and $y=\operatorname{dim} Y$. It suffices to show that the identity is a matrix from $K$ to $T_{i}$.

Let $C$ be a matrix from $K$ to $T_{i+1}$ with $|C| \notin T_{i+1}^{2}$. (This can be done since $N\left(K / T_{i+1}\right) \nsubseteq T_{i+1}^{2}$. $) \quad$ We have $C \sim I \oplus-1 \oplus s \oplus t$ where $s, t \in T_{i+1}$ by $[5,63: 17]$. Let $e \in T_{i}$ be such that $T_{i+1}=T_{i}(\sqrt{e})$. Let $M=\left\|\frac{1}{\sqrt{e}}-\sqrt{\bar{e}}\right\|$ and $E(q)=M D(q) M^{\prime}$ for $q \in T_{i+1}$. We have that

$$
S(r)=(I \oplus-1) \otimes E(r) \oplus E(r s) \oplus E(r t)
$$

is a matrix from $K$ to $T_{i}$ for nonzero $r \in T_{i+1}$. By (iii) we have $|S(r)| \in T_{i}^{2}$. Since

$$
\operatorname{dim}(I \oplus-1)=\operatorname{dim} S(r) / 2-2 \equiv 2(\bmod 4) \text { by (i) , }
$$

we have

$$
|(I \oplus-1) \otimes E(r)| \in T_{i}^{2} .
$$

Hence $|E(r s)| \in|E(r t)| \cdot T_{i}^{2}$. Thus 


$$
\begin{aligned}
c(S(r)) & =c((I \oplus-1) \otimes E(r)) c(E(r s)) c(E(r t))(|E(r s)|,-1) \\
& =(-1,-1) c(E(r s)) c(E(r t))(|E(r s)|,-1) \text { by }\left(^{*}\right) .
\end{aligned}
$$

Any $q \in T_{i+1}$ has the form $a+b \sqrt{e}$ with $a, b \in T_{i}$. Write $q_{1}=a$. If $q_{1} \neq 0$, then

$$
c(E(q))=\left(2 q_{1},-|E(q)|\right)(-1,|E(q)|) .
$$

If $(r s)_{1}(r t)_{1} \neq 0$, we have

$$
c(S(r))=\left(-(r s)_{1}(r t)_{1},-|E(r s)|\right) .
$$

We may choose $r=s^{-1}(l+\sqrt{\bar{e}})^{2} \sqrt{\bar{e}}$ with $l=0,1$, or 4 such that $(r s)_{1}(r t)_{1} \neq 0$. Since $-|E(r s)| \in T_{i}^{2}$, we have $c(S(r))=+1$.

Lemma 5. If $\sum_{1}^{m}\left[K_{i}: L\right]$ is odd, the identity is a matrix from $\sum_{1}^{m} \oplus K_{i}$ to $L$.

Proof. By Lemmas 3 and 4 we are done unless $\left[K_{i}: L\right]=d$ is even and $N\left(K_{i} / L\right) \nsubseteq L^{2}$ for some $i$. Suppose that this is the case. Since $N\left(K_{i} / L\right) \nsubseteq L^{2}$, there is a matrix $B$ from $K_{i}$ to $L$ such that $(-1)^{d / 2}|B| \notin L^{2}$. Let $C$ be a matrix from $\Sigma_{j \neq i} \oplus K_{j}$ to $L$. Let

$$
A=|B| \cdot|C| \cdot C \oplus a B
$$

where $a \in L$ is chosen so that

$$
c(A)=c(|B| \cdot|C| \cdot C)(|B|,-1) c(B)\left(a,(-1)^{d / 2}|B|\right)=+1 .
$$

Lemma 6. If $\Sigma_{1}^{m}\left[K_{i}: L\right]$ is even, $N\left(K_{1} / L\right) \nsubseteq L^{2}$, and $k$ is a rational integer, then there is a matrix $A$ from $\Sigma_{1}^{m} \oplus K_{i}$ to $L$ such that $c(A)(|A|,-1)^{k}=+1$.

Proof. Let $B$ be a matrix from $\Sigma_{1}^{m} \oplus K_{i}$ to $L$ such that $(-1)^{n}|B| \notin L^{2}$ where $n=\sum_{1}^{m}\left[K_{i}: L\right] / 2$. Let $A=a B$ where $a \in L$ is chosen so that $c(A)(|A|,-1)^{k}=c(B)(|B|,-1)^{k}\left(a,(-1)^{n}|B|\right)=+1$.

6. Proof of Theorem 2. If $n$ if odd, apply Lemmas 2 and 5 . Let $B$ be the matrix given by Theorem 3. Define $A=|B| \cdot B$. If $n$ is even, apply Lemmas $2,3,4$ and 6 . Let $A$ be the matrix given by Theorem 3. In both cases, behavior at the exceptional spot is handled by the Hilbert reciprocity formula [5, p. 190].

7. Matrices with given roots. We prove

Theorem 4. Let $F$ be an algebraic number field. Let $\theta$ be the root of an irreducible F-polynomial $q$ of degree $n$. Then $\theta$ is the 
characteristic root of some symmetric F-matrix if and only if $q$ is F-real. When such a matrix exists, it may be chosen to have dimension $n$ or $n+1$, whichever is odd. This dimension is the least possible

(1) if $n$ is odd or

(2) if $n \equiv 2(\bmod 4)$ and $(-1) \notin N(F(\theta) / F) \cdot F^{2}$.

Proof. Use Theorem 1 with $p(x)=q(x)$ or $x q(x)$. The result is clearly best possible when $n$ is odd. Suppose $n \equiv 2(4)$ and $n$ is least possible. Let $\alpha_{i}=\theta^{i-1}$ and $M=\left\|\alpha_{i}^{(j)}\right\|$. By the converse of Lemma 1 when $p$ does not have repeated roots (see [6, Lemma 1.1] for a proof), there is an $F$-matrix $T$ and a $\lambda \in F(\theta)$ such that $M D(\lambda) M^{\prime}=T T^{\prime}$. Noting that $\left|M M^{\prime}\right|=-N\left(p^{\prime}(\theta)\right)$, we get

$$
-1 \in N(F(\theta) / F) \cdot F^{2} \text {. }
$$

By class field theory, for all $n \equiv 2(4)$ there exist $F$ and $\theta$ such that $n+1$ is the least possible dimension.

I would like to thank Drs. O. Taussky and E. C. Dade for their assistance.

\section{REFERENCES}

1. H. Hasse, Bericht Über neuere Untersuchungen und Probleme aus der Theorie der algebraischen Zahlkörper I, Jber. Deutsch. Math.-Verein. 35 (1926), 1-55.

2. — Zahlentheorie, 2nd ed., Akademie-Verlag, Berlian, 1963.

3. B. W. Jones, The arithmetic theory of quadratic forms, Carus Monograph v. 10, Math. Assn. Amer., 1950.

4. F. Krakowski, Eigenverte und Minimalpolynome symmetrischer Matrizen in kommutativen Körpern, Comment. Math. Helv. 32 (1958), 224-240.

5. O. T. O'Meara, Introduction to quadratic forms, Grund. Math. Wiss. v. 117, Academic Press Inc., New York, 1963.

6. A. P. Sapiro, Characteristic polynomials of symmetric matrices (Russian), Sibirsk. Mat. Z., 3 (1962), 280-291.

7. J.-P. Serre, Corps locaux, Hermann, Paris, 1962.

\section{HARVARD UNIVERSITY}

Received August 15, 1966. This extends a part of my doctoral dissertation written as a National Science Foundation fellow under Dr. O. Taussky at the California Institute of Technology. 



\title{
PACIFIC JOURNAL OF MATHEMATICS
}

\author{
EDITORS
}

\section{H. ROYDEN}

Stanford University

Stanford, California

\author{
J. P. JANS \\ University of Washington \\ Seattle, Washington 98105
}

\section{J. DugundJI}

Department of Mathematics

University of Southern California

Los Angeles, California 90007

\section{RICHARD ARENS}

University of California

Los Angeles, California 90024

\section{ASSOCIATE EDITORS}
E. F. BECKENBACH
B. H. NEUMANN
F. WOLF
K. YOSIDA

\section{SUPPORTING INSTITUTIONS}

\author{
UNIVERSITY OF BRITISH COLUMBIA \\ CALIFORNIA INSTITUTE OF TECHNOLOGY \\ UNIVERSITY OF CALIFORNIA \\ MONTANA STATE UNIVERSITY \\ UNIVERSITY OF NEVADA \\ NEW MEXICO STATE UNIVERSITY \\ OREGON STATE UNIVERSITY \\ UNIVERSITY OF OREGON \\ OSAKA UNIVERSITY \\ UNIVERSITY OF SOUTHERN CALIFORNIA
}

\author{
STANFORD UNIVERSITY \\ UNIVERSITY OF TOKYO \\ UNIVERSITY OF UTAH \\ WASHINGTON STATE UNIVERSITY \\ UNIVERSITY OF WASHINGTON \\ AMERICAN MATHEMATICAL SOCIETY \\ CHEVRON RESEARCH CORPORATION \\ TRW SYSTEMS \\ NAVAL WEAPONS CENTER
}

Mathematical papers intended for publication in the Pacific Journal of Mathematics should be in typed form or offset-reproduced, double spaced with large margins. Underline Greek letters in red, German in green, and script in blue. The first paragraph or two must be capable of being used separately as a synopsis of the entire paper. It should not contain references to the bibliography. Manuscripts, in duplicate if possible, may be sent to any one of the four editors. All other communications to the editors should be addressed to the managing editor, Richard Arens, University of California, Los Angeles, California 90024.

Each author of each article receives 50 reprints free of charge; additional copies may be obtained at cost in multiples of 50 .

The Pacific Journal of Mathematics is published monthly. Effective with Volume 16 the price per volume (3 numbers) is $\$ 8.00$; single issues, $\$ 3.00$. Special price for current issues to individual faculty members of supporting institutions and to individual members of the American Mathematical Society: $\$ 4.00$ per volume; single issues $\$ 1.50$. Back numbers are available.

Subscriptions, orders for back numbers, and changes of address should be sent to Pacific Journal of Mathematics, 103 Highland Boulevard, Berkeley 8, California.

Printed at Kokusai Bunken Insatsusha (International Academic Printing Co., Ltd.), 7-17, Fujimi 2-chome, Chiyoda-ku, Tokyo, Japan.

PUBLISHED BY PACIFIC JOURNAL OF MATHEMATICS, A NON-PROFIT CORPORATION

The Supporting Institutions listed above contribute to the cost of publication of this Journal, but they are not owners of publishers and have no responsibility for its content or policies. 


\section{Pacific Journal of Mathematics}

\section{Vol. 25, No. $3 \quad$ November, 1968}

Philip Marshall Anselone and Theodore Windle Palmer, Collectively

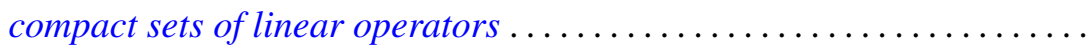

Philip Marshall Anselone and Theodore Windle Palmer, Spectral analysis of collectively compact, strongly convergent operator sequences.........

Edward A. Bender, Characteristic polynomials of symmetric matrices...... 433

Robert Morgan Brooks, The structure space of a commutative locally convex

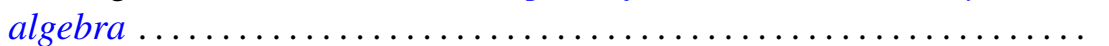

Jacob Feldman and Frederick Paul Greenleaf, Existence of Borel

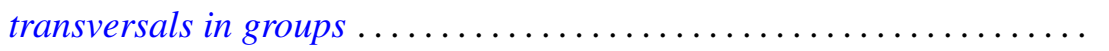

Thomas Muirhead Flett, Mean values of power series 463

Richard Vernon Fuller, Relations among continuous and various

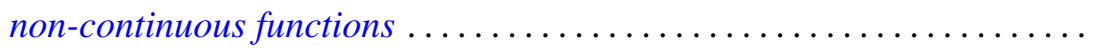

Philip Hartman, Convex sets and the bounded slope condition ............

Marcel Herzog, On finite groups containing a CCT-subgroup with a cyclic Sylow subgroup .

James Secord Howland, On the essential spectrum of Schroedinger

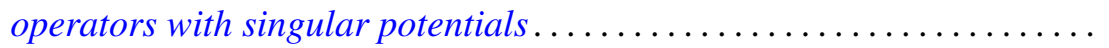

Thomas William Hungerford, On the structure of principal ideal rings .....

Paul Joseph Kelly and Ernst Gabor Straus, Curvature in Hilbert geometries. II mpati Madhusudana Rao, Linear functionals on Orlicz spaces:

Malempati Madhusudana Rao, Linear functionals on Orli
$\quad$ General theory............................
Stanley F. Robinson, Theorems on Brewer sums.......
Ralph Tyrrell Rockafellar, A general correspondence betw

Malempati Madhusudana Rao, Linear functiona
$\quad$ General theory.........................
Stanley F. Robinson, Theorems on Brewer sums
Ralph Tyrrell Rockafellar, A general corresponden

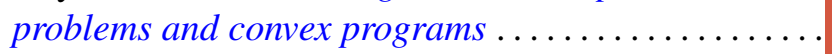

Richard Benjamin Sher, Defining subsets of $E^{3}$ by cubes.

Howard Jacob Weiner, Invariant measures and Cesàro summability... 